\title{
SIGNIFICADO DO ELETRENCEFALOGRAMA NO DIAGNÓSTICO DE CRISE CONVULSIVA ÚNICA EM CRIANÇAS
}

\author{
Rubens Moura Ribeiro* \\ JORGE ARMBRUST-FigUeIREDO ** \\ VAleriana Moura Ribeiro*
}

Os trabalhos experimentais e a clínica demonstram que uma crise convulsiva isolada pode determinar lesão cerebral mesmo em crianças e, por esta razão, todo paciente que tenha manifestado uma crise convulsiva deverá ser examinado minuciosamente ${ }^{4,13}$. Um dos problemas fundamentais é estabelecer a existência e o tipo de comprometimento do sistema nervoso central, quer pelo exame clínico, quer mediante exames subsidiários. De acôrdo com a literatura, o registro eletrencefalográfico representa um método útil que pode confirmar, ou não, a presença de algum fator orgânico na etiologia da crise convulsiva. Evidentemente, a presença de lesão cerebral poderá ser melhor demonstrada pela associação dos achados do exame neurológico às alterações eletrencefalográficas. Entretanto, a freqüente ausência de sinais neurológicos em pacientes portadores de crises convulsivas, bem como a dificuldade na realização do exame neurológico completo em crianças, justifica o emprêgo de exames subsidiários com a finalidade de evidenciar possiveis alterações cerebrais decorrentes ou responsáveis pelas crises convulsivas.

O presente trabalho tem o intuito de avaliar o valor do eletrencefalograma (EEG) em pacientes portadores de crise convulsiva única até a ocasião da feitura do exame, e verificar se, nessas condições, já ocorreram alterações que possam estar relacionadas com processos orgânicos encefálicos bem definidos.

\section{MATERIAL, MÉTODO E RESULTADOS}

O material consta de 20 pacientes cujas idades variaram entre um e 14 anos, sendo 15 do sexo masculino e 5 do feminino. As crises apresentadas pelos pacientes puderam ser agrupadas nos seguintes tipos clínicos: crise tipo grande mal ( 8 casos), crise lateralizada à direita (6), crise lateralizada à esquerda (3), crise psicomotora (1), crise controversiva (1) e crise tipo pequeno mal (1). Esses pacientes foram seguidos, do ponto de vista clínico, por um periodo de até 3 anos, com o intuito de se registrar eventual recorrencia da crise.

Os registros eletrencefalográficos foram feitos em aparelho Grass de 8 canais, colocando os eletrodos segundo a técnica usual internacional. Em 12 pacientes obtivemos a ativação pela hiperpnéia e em 8 registramos, também, o traçado durante o sono induzido por barbitúricos.

Trabalho da Clínica Neurológica da Faculdade de Medicina de Ribeirão Prêto, da Universidade de São Paulo: * Assistente; ** Professor. 
Os traçados eletrencefalográficos foram normais em 7 casos (35\%) e registraram alguma forma de alteração em $13(65 \%)$, sendo que, já durante o tracado de repouso, 8 pacientes evidenciaram EEG com ritmo de base irregular (Quadro 1).

As alterações eletrencefalográficas podem ser resumidas da seguinte maneira: irregularidade difusa do ritmo de base em 8 casos (40\%); disritmia paroxistica por onda tipo "sharp" em 12 pacientes $(60 \%)$; disritmia paroxistica por onda espicula de 3 ciclos por segundo, em um caso $(5 \%)$.

A incidência topográfica das disritmias paroxisticas obedeceu à seguinte distribuição: foco fronto-temporal esquerdo (6 casos); foco temporal médio esquerdo (3); foco fronto-temporal direito (2); foco parieto-occipital esquerdo (1); região centrencefálica (1).

$\mathrm{Na}$ evolução clinica dêsses casos verificamos que 8 (40\%) apresentaram crises convulsivas recorrentes.

\begin{tabular}{|c|c|c|c|c|c|c|c|c|}
\hline Caso & Nome & $E E G$ & Sexo & $\begin{array}{c}\text { Idade } \\
\text { (anos) }\end{array}$ & Resultado & $d o$ & $E E G$ & \\
\hline 1 & MJRG & 188 & $\mathbf{F}$ & 7 & Irregularidade no & ritmo & + & "sharp" \\
\hline 2 & JBR & 248 & $\mathbf{M}$ & 5 & Irregularidade no & ritmo & + & "sharp" \\
\hline 3 & RLP & 255 & $\mathbf{M}$ & 10 & Onda "sharp" & & & \\
\hline 4 & JRB & 356 & $\mathbf{M}$ & 14 & Onda "sharp" & & & \\
\hline 5 & AVL & 383 & M & 13 & Onda "sharp" & & & \\
\hline 6 & $\mathrm{JL}$ & 403 & M & 7 & Irregularidade no & ritmo & + & "sharp" \\
\hline 7 & PGJ & 573 & $\mathbf{M}$ & 4 & Normal & & & \\
\hline 8 & GAR & 574 & $\mathbf{M}$ & 5 & 3 ciclos por segur & ndo & & \\
\hline 9 & SHJ & 560 & $\mathbf{M}$ & 1,7 & Normal & & & \\
\hline 10 & HLO & 597 & $\mathbf{M}$ & 14 & Normal & & & \\
\hline 11 & AMAP & 603 & $\mathbf{F}$ & 13 & Irregularidade no & ritmo & + & "sharp" \\
\hline 12 & ASP & 670 & $\mathbf{M}$ & 4 & Normal & & & \\
\hline $\mathbf{1 3}$ & MMSC & 932 & $\mathbf{F}$ & 13 & Irregularidade no & ritmo & + & "sharp" \\
\hline 14 & MSP & 940 & M & 8 & Irregularidade no & ritmo & & \\
\hline 15 & OMN & 1063 & $F$ & 7 & Onda "sharp" & & & \\
\hline 16 & AAR & 1223 & M & 2,8 & Irregularidade no & ritmo & + & "sharp" \\
\hline 17 & JRJF & 1254 & $\mathbf{M}$ & 3 & Normal & & & \\
\hline 18 & MFM & 1292 & $\mathbf{F}$ & 9 & Irregularidade no & ritmo & + & "sharp" \\
\hline 19 & $\mathrm{DC}$ & 1397 & M & 13 & Normal & & & \\
\hline 20 & JSFS & 1456 & M & 2 & Normal & & & \\
\hline
\end{tabular}

Quadro 1 - Resultados eletrencefalográficos nos 20 pacientes.

\section{COMENTARIOS}

A incidência de crise convulsiva única em crianças é de 6 a $7 \%$ segundo estatísticas de Baird ${ }^{2}$ e Breg ${ }^{3}$. Do ponto de vista prático, o problema adquire importância quando tentamos avaliar o prognóstico dos casos em que a primeira crise convulsiva ocorreu no decorrer da primeira infância.

Tôda crise convulsiva é um sintoma que indica uma alteração neuronal cerebral; esta alteração pode decorrer de uma lesão orgânica encefálica, ou indicar apenas um distúrbio funcional temporário. A idade em que a crise convulsiva se manifesta representa um fator importante na avaliação diagnóstica e prognóstica. Assim, uma crise convulsiva que se manifesta pela primeira vez até os dois anos de idade, pode ser a primeira 
manifestação de alguma alteração cerebral, em geral associada a trauma de parto ou a defeitos congênitos ${ }^{10}$, ao passo que as crises convulsivas de causa desconhecida ocorrem, em geral, pela primeira vez em crianças entre 2 e 10 anos de idade ${ }^{4}$.

Representa, pois, a eletrencefalografia método útil e prático para o diagnóstico e prognóstico de uma crise convulsiva, já que anormalidades eletrencefalográficas podem existir mesmo na ausência de crise clínica $6,7,9,12$. Evidentemente, o EEG normal de uma criança que tenha sofrido crise convulsiva permite melhor prognóstico do que um EEG alterado 5, 8. Entretanto, um EEG negativo não exclui o diagnóstico de epilepsia, sendo, por vêzes, necessária a repetição do exame para a sua confirmação. Aass e col. ${ }^{1}$ demonstraram que a presença de anormalidades eletrencefalográficas em pacientes que tiveram crise única aumenta a possibilidade de desenvolvimento de crises recorrentes.

$\mathrm{Na}$ análise de nossos resultados verificamos que em 13 pacientes $(65 \%)$, o traçado de repouso ou ativado pela hiperpnéia evidenciava a presença de disritmia paroxística por onda "sharp", isolada ou associada a um rítmo de base irregular sugestivo de um fator lesional preexistente.

No seguimento dêsses pacientes durante três anos verificamos que os $\delta$ casos $(40 \%)$ em que houve recorrência da crise convulsiva eram exatamente aquêles que apresentavam EEG com ritmo de base irregular, associado à presença de onda "sharp". Infelizmente não fizemos o seguimento eletrencefalográfico dêsses pacientes e, assim, não pudemos avaliar as eventuais modificações de seus traçados. Por outro lado, deve-se ter em mente que uma relação entre os achados clínicos e o EEG não pode ser absoluta, já que a presença de uma atividade paroxística no exame inicial, não significa necessàriamente um prognóstico pior.

\section{RESUMO}

Foram estudadas, do ponto de vista eletrencefalográfico, 20 crianças que apresentaram crise convulsiva única, com intuito de verificar se nessas condições já ocorrem alterações que possam estar relacionadas com processos orgânicos encefálicos. Considerado de maneira global, o EEG mostrou-se alterado em 13 casos $(65 \%)$ e foi normal em $7(35 \%)$; as alterações foram do tipo focal em 12 casos e difusas em um. Os eletrencefalogramas com ritmo de base irregular e disritmia paroxística registrada durante a ativação pela hiperpnéia ( 8 casos ou $40 \%$ ) corresponderam aos pacientes que, ulteriormente, apresentaram recorrência da convulsão.

\section{SUMMARY}

The value of the EEG in the diagnosis and prognosis of a single convulsive disorder in children.

The authors studied the EEG of 20 children with history of a single epileptic seizure, in order to establish a presumptive pathological factor 
in the central nervous system. In this series, 13 patients $(65 \%)$ had abnormal tracings, and $7(35 \%)$ had normal EEG records. The most frequent abnormality found was a focal change, recorded in 12 patients, while the diffuse type was found in just one. Abnormal tracings, either at rest or during overventilation, were most frequently seen in 8 patients which had recurrent seizures, registered in a three years follow-up.

\section{REFERENCIAS}

1. AASS, F.; KAADA, B. R. \& TORP, K. H. - The diagnosis and prognostic value of the initial electroencephalogram in children with convulsions. Acta paediat. 45:335-342, 1956. 2. BAIRD, H. W. - Convulsions in infancy and childhood. Pediat. Clin. N. Amer. 10:705-721, 1963. 3. BREG, W. R. \& YANAET, H. - The child in a convulsion. Pediat. Clin. N. Amer. 9:101-112, 1962. 4. CARTER, S. - Diagnosis and treatment: management of the child who has had one convulsion. Pediatrics, 33:431-434, 1964. 5. CHAO, D. H.-C.; DRUCKMAN, R. \& KELLAWAY, P. - Convulsive Disorders of Children. W. B. Saunders Co., Philadelphia, 1958. 6. FOIS, A. - Clinical Electroencephalography in Epilepsy and Related Conditions in Children. Charles C. Thomas, Springfield (Illinois), 1963. 7. GIBBS, F. A. \& GIBBS, E. L. - Atlas of Electroencephalography, vol. 1. Addison-Wesley, Cambridge (Mass), 1958. 8. HARRIS, R. \& AIZARD, J. P. M. - The electroencephalogram in neonatal convulsions. J. Pediat. 57:501-520, 1960. 9. HUGHES, R. R. - An Introduction to Clinical Electroencephalography. John Wright and Sons, Bristol, 1961. 10. JUUL-JENSEN, P. - Epilepsy: a clinical and social analysis of 1020 adult patients with epileptic seizures. Acta Neurol. Scandinavica, supl. 5, 1964. 11. KEITH, H. M. - Convulsive Disorders in Children. Little Brown Co., Boston, 1963. 12. KILOH, L. G. \& OSSELTON, J. W. - Clinical Electroencephalography. Butterworths, London, 1961. 13. ZIMMERMAN, H. M. - The basis of convulsive attacks in children: an experimental study. Bull. N.Y. Acad. Med. 35: 801-809, 1959.

Clinica Neurológica - Faculdade de Medicina - Ribeirão Prêto, SP - Brasil. 